\title{
Expansión de las ciudades en Centroamérica y generación de riesgos urbanos
}

\author{
- Mario Lungo*
}

\section{Introducción: problema antiguo, problemas nuevos}

La expansión territorial de las ciudades grandes en América Latina no es fenómeno nuevo, pero presenta sensibles diferencias con las características que éste ha asumido particularmente en los Estados Unidos.

En efecto, si la expansión urbana en este último país ha configurado un paisaje donde los conjuntos residenciales para clases medias y altas son vinculados por una red de autopistas destinadas para los automóviles privados, principalmente, en América Latina este territorio ha sido, en la mayoría de los casos, el reino de la marginalidad y la precariedad de la llamada ciudad ilegal de los pobres.

Sin embargo, esta situación ha estado cambiando durante los últimos años; encontramos la construcción de conjuntos habitacionales para sectores de bajos y medios ingresos, grandes centros comerciales y exclusivas residenciales "cerradas", en una abiganada mezcla que está modificando el antiguo patrón de segregación socio-espacial.

Evidentemente, hay muchas diferencias y particularidades entre las grandes ciudades de la región, particularmente las áreas metropolitanas, derivadas de la

* Trabajo elaborado para la reunión de CRIES sobre "Gestion de riesgos y vulnerabilidad en el Gran Caribe", La Habana, febrero de 2001. 
historia y la geografía, pero lo que queremos destacar aquí es el panorama predominante para efectos del objetivo de este trabajo.

El fenómeno a que nos estamos refiriendo es antiguo. Data, en la mayoría de países, de principios del siglo $\mathrm{XX}$, cuando muchas ciudades conocieron una dinámica expansión poblacional, económica y territorial. Dos ejemplos muy alejados permiten ilustrar lo anterior: el primero, São Paulo, en Brasil, que pasa de tener 47,697 habitantes en 1886, a alcanzar cerca de 17 millones al finalizar el siglo (Rolnik, 1997); el segundo, San Salvador, en El Salvador, que crece de 50,304 habitantes en 1905, a un poco más de dos millones en el año 200() (Lungo y Baires, 1988). En ambos casos, la producción cafetalera es la base de partida de esta expansión. El desarrollo industrial y financiero posterior, y la dimensión del país, explica la explosión demográfica de la primera.

Y las dos ciudades, en diferente dimensión, se verán enfrentadas durante las últimas décadas del siglo XX a un nuevo desafío: el surgimiento de inéditos riesgos urbanos antes no imaginados y para los cuales las ciudades, sus habitantes y los responsables de la gestión de su desarrollo no estaban preparados.

Este trabajo trata de hacer una reflexión sobre los nuevos riegos urbanos que se están gestando por la expansión territorial de las principales ciudades centroamericanas durante los últimos años. Para esto, se presentan, en el segundo punto, los rasgos principales que caracterizan estos procesos de expansión urbana en San Salvador, San José y Panamá, proponiendo posteriormente una serie de indicadores que deberían ser construidos para su análisis en profundidad. Para esto último, se hace una revisión crítica de los indicadores urbanos propuestos por HABITAT/PNUD, los que, en nuestra opinión, no incorporan plenamente esta dimensión de la realidad urbana.

En el tercer punto se intenta avanzar analíticamente en la construcción de riesgos urbanos, a partir de una serie de vulnerabilidades claves planteadas en importantes trabajos (Fernández, 1996; Lavell, 200()). Este ensayo metodológico es de carácter exploratorio y tiene como objetivo sentar las bases para el análisis de casos concretos.

El cuarto punto consiste en la aplicación preliminar del camino esbozado en el punto anterior al caso del Área Metropolitana de San Salvador, ciudad que se enfrenta a importantes riesgos ambientales derivados de una inadecuada utilización y modificación del territorio urbanizado, de la acentuada pobreza y segregación socio-espacial, y de las deficiencias de la gestión urbana.

En el punto final, se discute el papel de los gobiernos locales y las organizaciones ciudadanas en la gestión de los riesgos urbanos.

Se trata, en síntesis, de discutir la relación entre un problema antiguo, la expansión territorial de las ciudades, y un problema nuevo, la emergencia de riesgos 
urbanos no incorporada anteriormente en los estudios sobre el desarrollo y en la planificación de las ciudades.

Esta situación no es exclusiva de las ciudades de los países en desarrollo. En el caso de una de las grandes aglomeraciones del mundo desarrollado, Los Ángeles, en California, a pesar de estar situada en una región donde están presentes desde hace milenios amenazas naturales de gran envergadura, y ser una de las ciudades más estudiadas desde diversos aspectos, es solamente en años recientes que la generación de riesgos urbanos y su relación con la forma de crecimiento territorial se ha colocado en el centro de las preocupaciones de muchos académicos y responsables del desarrollo (Davis, 1998).

Este trabajo se apoya en distintas investigaciones realizadas durante los años anteriores, tanto en el ámbito del desarrollo y la gestión urbana, como en el campo de los desastres, y en la riqueza de los aportes del conjunto de trabajos de la Red de Estudios Sociales en Prevención de Desastres en América Latina, que ha venido realizando estudios innovadores en este campo.

2. Características de la expansión de las ciudades San Salvador, San José y Panamá, al iniciarse el siglo XXI

\section{MAPA 1}

Principales ciudades centroamericanas

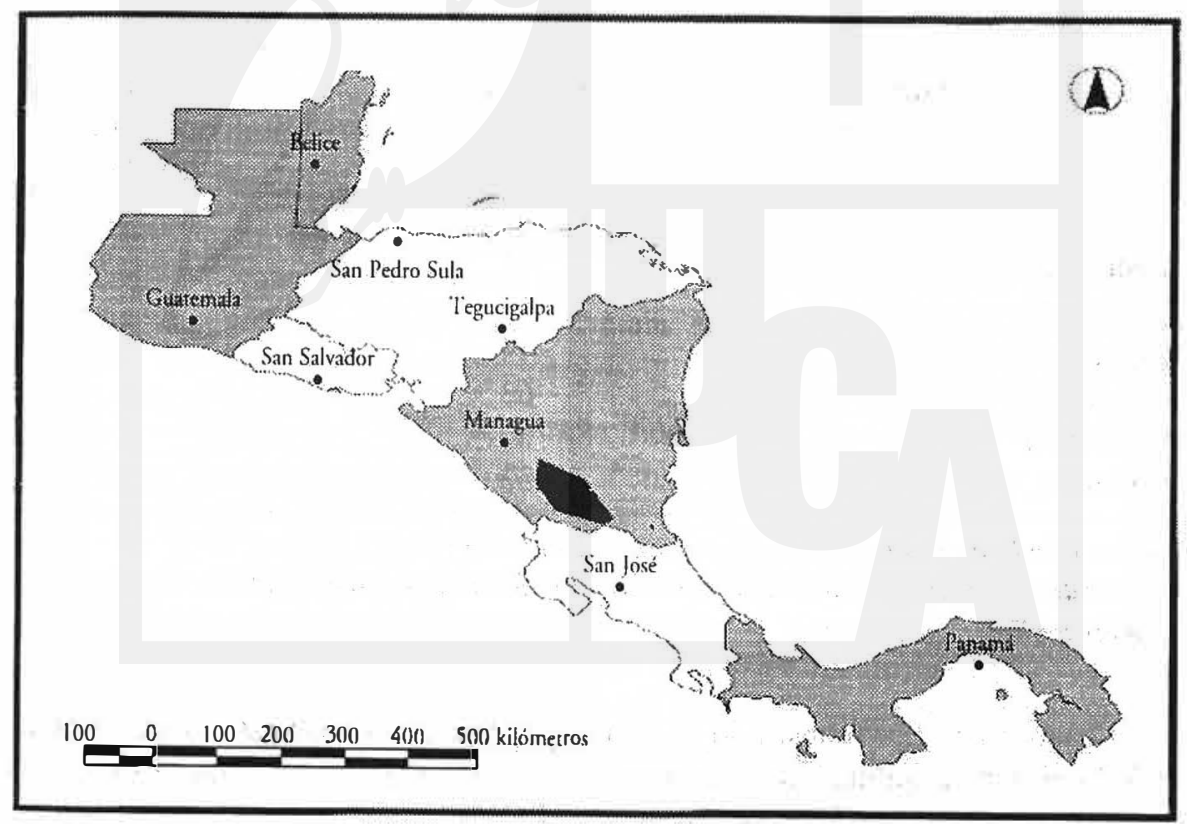

Expansión de las ciudades en Centroamérica 
La mayor parte de las principales ciudades centroamericanas, que se convirtieron en las capitales de las repúblicas centroamericanas después de la independencia de 1821, se localizaron en las zonas interiores de los territorios colonizados. La excepción fue Panamá, construida desde el inicio a la orilla del mar como un punto clave del comercio hacia América del Sur. Estas ciudades cumplían funciones económicas secundarias vinculadas a sus ámbitos económicos regionales.

Con la introducción de las economías cafetaleras durante la segunda mitad del siglo XIX y la instalación de las primeras industrias manufactureras en las primeras décadas del siglo siguiente, algunas de estas ciudades (San Salvador, San José y Managua), se consolidaron como las principales ciudades centroamericanas junto a Guatemala, que había sido la capital colonial, y Panamá. La diferencia la marca Honduras, donde, aunque la principal concentración urbana era Tegucigalpa, vinculada a las antiguas explotaciones mineras de la zona, va a competir, desde la segunda mitad del siglo XX, con San Pedro Sula, situada cerca de la costa caribeña, que se convertirá en el centro industrial del país.

Estas ciudades, conformadas al finalizar el siglo XX en áreas metropolitanas, al expandirse ternitorialmente, albergan una importante proporción de la población urbana de los distintos países:

Cuadro 1

Centroamérica: Población total, población urbana y población del área metropolitana principal (1999)

\begin{tabular}{lccc}
\hline País & $\begin{array}{c}\text { Población total } \\
\text { (millones) }\end{array}$ & $\begin{array}{c}\text { Población urbana } \\
(\%)\end{array}$ & $\begin{array}{c}\text { Población del } \\
\text { área metropolitana } \\
\text { principal (a) (\%) }\end{array}$ \\
\hline Guatemala & 11.1 & 41 & 20 \\
El Salvador & 6.2 & 45 & 33 \\
Honduras & 6.3 & 44 & 15 \\
Nicaragua & 4.9 & 63 & 30 \\
Costa Rica & 3.9 & 50 & 38 \\
Panamá & 2.8 & 53 & 41 \\
\hline
\end{tabular}

Fuente: Fondo de Población de las Naciones Unidas (FNUAP)

(a) Cálculos propios a partir del ajuste de los territorios comprendidos en las áreas metropolitanas principales.

El cuadro anterior muestra lo afirmado, a pesar de que las estadísticas oficiales subvaloran la población urbana real, en casos como los de San Salvador o San José, por los criterios censales adoptados. 
Por otra parte, estamos ante una situación peculiar que agrega peso a estas ciudades: son las principales aglomeraciones urbanas en países de limitada extensión territorial. En otros términos, se podría decir que se trata de ciudades grandes en países pequeños:

\section{Cuadro 2}

\section{Centroamérica: Extensión territorial y población del área metropolitana principal}

\begin{tabular}{|l|c|c|}
\hline País & $\begin{array}{c}\text { Extensión territorial } \\
\left(\mathbf{K ~ m}^{\mathbf{2}}\right)\end{array}$ & $\begin{array}{c}\text { Población principal } \\
\text { área metropolitana }\end{array}$ \\
\hline Guatemala & 108,800 & $2,200,000$ \\
El Salvador & 20,000 & $2,050,000$ \\
Honduras & 112,000 & 950,000 \\
Nicaragua & 139,000 & $1,500,000$ \\
Costa Rica & 51,100 & $1,500,000$ \\
Panamá & 77,000 & $1,150,000$ \\
\hline
\end{tabular}

FUENTE: FNUAP y cálculos propios.

Adicionalmente, dentro de las redes urbanas nacionales, estas ciudades presentan índices de primacía elevados, con la excepción del caso de Honduras, antes mencionado:

Para efectos de la problemática que nos ocupa - la expansión territorial de las ciudades y la generación de riesgos urbanos-, es importante señalar que la mayoría de estas ciudades se encuentra en la franja volcánica de alta actividad sísmica y en ámbitos ecológicos de gran valor, por lo que el impacto negativo de la expansión urbana es muy elevado, tanto en términos de afectación de los recursos como en la generación de riesgos.

Para analizar las características específicas de la expansión territorial de las principales ciudades centroamericanas, hemos seleccionado tres de ellas: San Salvador, San José y Panamá.

Las dos primeras se encuentran en ámbitos ecológicos similares: valles intermontanos afectados por erupciones volcánicas y sismos frecuentes; tierras de alta fertilidad y gran biodiversidad. Su expansión territorial se ha realizado provocando la disminución de algunas de las tierras de mayor productividad agrícola, afectando la flora y la fauna y ocupando zonas de alto riesgos. Panamá presenta menores amenazas sísmicas y no se encuentra en una zona volcánica, existiendo solamente aquellas asociadas a su posición costera, aunque, por estar 


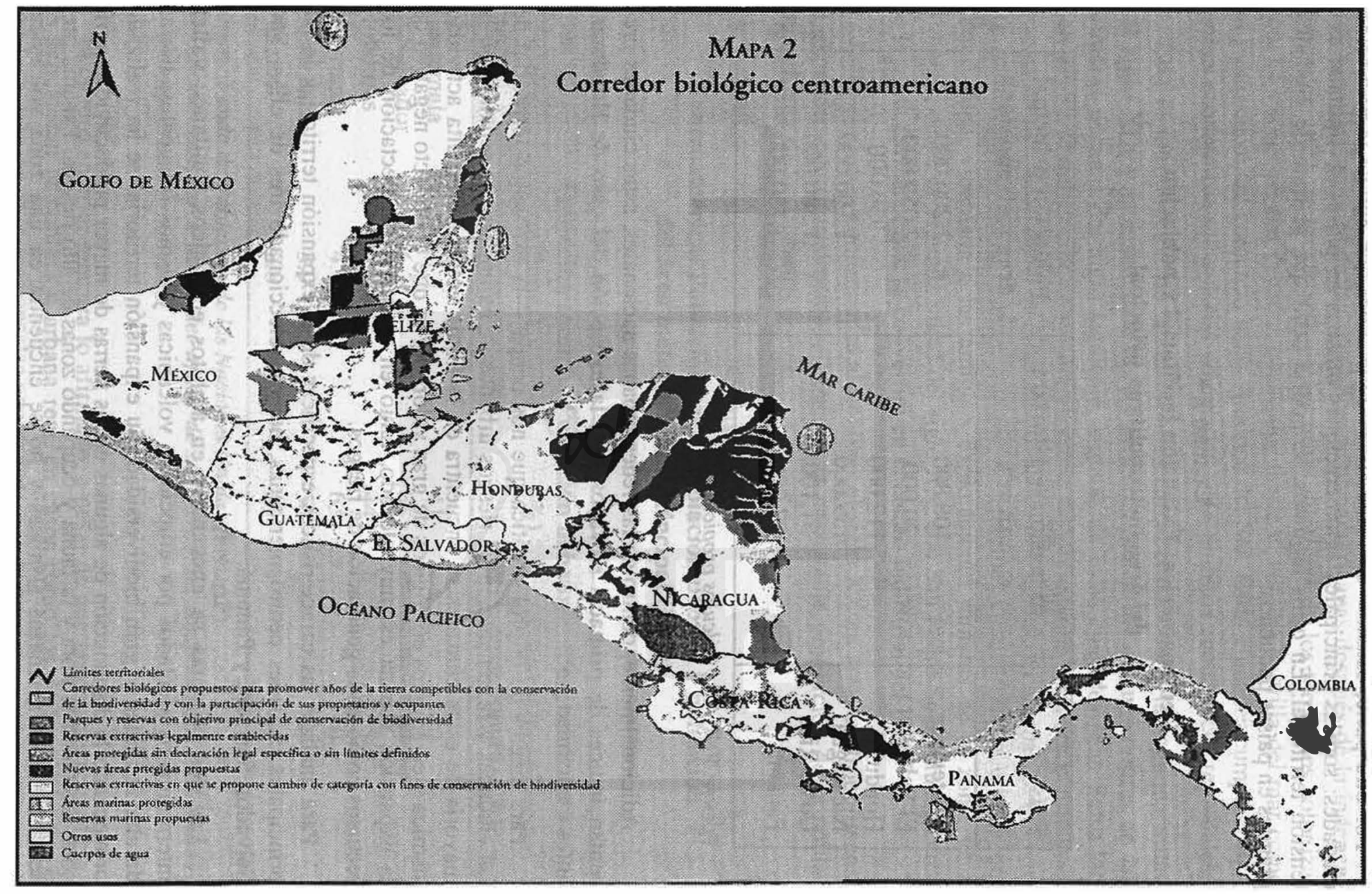

Digitalizado por Biblioteca "P. Florentino Idoate, S.J." Universidad Centroamericana José Simeón Cañas 
Cuadro 3

Centroamérica: Relación entre la población de la capital (áreas metropolitanas) y la segunda ciudad de cada país

\begin{tabular}{|l|c|c|c|c|}
\hline País & $\mathbf{1 9 6 0}$ & $\mathbf{1 9 7 0}$ & $\mathbf{1 9 8 0}$ & $\mathbf{1 9 9 5}$ \\
\hline Guatemala & 13.7 & 16.1 & 23.2 & 16.6 \\
El Salvador & 4.8 & 5.9 & 6.5 & 10.0 \\
Honduras & 2.3 & 1.8 & 1.7 & 1.5 \\
Nicaragua & 5.2 & 7.2 & 8.7 & 8.0 \\
Costa Rica & 10.3 & 11.9 & 15.0 & 13.0 \\
Panamá & 4.8 & 5.9 & 6.0 & 5.0 \\
\hline
\end{tabular}

Fut:Nite: Nuhn, Helmut: Estructura y desarrollo del sistema de ciudades de Centroamerica y Panamá, en Desarrollo polarizado de descentralización en América Central: El caso de Panamá, H. Nuhn y A. McKay, Hamburg, 1990, para los años de 1960 y 1970, excepto para San José en 1970, por cambios en la circunscripción; escimaciones propias a partir de cifras oficiales para 1980 y 1995.

ubicada sobre el Océano Pacífico, está menos expuesta a los frecuentes huracanes del Caribe.

Si en los casos de San Salvador y San José los límites a su expansión territorial son las zonas montañosas que las rodean, en el caso de Panamá, dicha expansión se ha visto radicalmente restringida por un hecho antrópico: la creación de la Zona del Canal como área a no urbanizar, indispensable para el funcionamiento del canal y para garantizar la seguridad militar del mismo. Por esta razón, el crecimiento físico de esta ciudad asumió una forma lineal que ha llevado a darle el calificativo de ciudad estrangulada (Uribe, 1989).

El Área Metropolitana de San Salvador (AMSS), tenía una población aproximada de 1.5 millones de habitantes en 1995, creció a dos millones en el año 2000 y se estima que alcanzará 2.5 millones en el 2010. La extensión era en el primero de estos años de $543.3 \mathrm{~km}^{2}$ (13 municipios), de los cuales estaban urbanizados 162.97 $\mathrm{km}^{2}$ (Umaña, 1995). Esta superficie se ha incrementado al agregarse al AMSS el municipio de Tonacatepeque, que es predominantemente rural. A pesar de la alta densidad demográfica del país y la ciudad, el patrón de construcción muestra una baja densidad en términos edificatorios. Sin embargo, el principal problema es la discontinuidad y la utilización de zonas de alto riesgo, tanto para la construcción de viviendas para los sectores, tanto de bajos como de altos ingresos. Lo sucedido a raíz del reciente terremoto del 13 de enero del 2001 es una comprobación muy clara de lo anterior. 


\section{Cuadro 4}

Incidencia de la expansión territorial

\begin{tabular}{|c|c|c|c|}
\hline Procesos & $\begin{array}{c}\text { San } \\
\text { Salvador }\end{array}$ & San José & Panamá \\
\hline 1. Pérdida de tierras agrícolas & + & + & - \\
\hline 2. Disminución de espacios verdes & + & + & +- \\
\hline $\begin{array}{l}\text { 3. Abandono y deterioro de áreas } \\
\text { centrales de la ciudad }\end{array}$ & + & +- & +- \\
\hline 4. Contaminación del aire & + & + & + \\
\hline $\begin{array}{l}\text { 5. Contaminación y reducción de } \\
\text { mantos acuíferos }\end{array}$ & + & +- & +- \\
\hline $\begin{array}{l}\text { 6. Extensión de trayectos entre residencia } \\
\text { y trabajo }\end{array}$ & + & + & + \\
\hline 7. Dispersión de los lugares de trabajo & + & + & + \\
\hline 8. Aumento de la segregación socio-espacial & +- & +- & + \\
\hline $\begin{array}{l}\text { 9. Incremento de los costos de la } \\
\text { infraestructura y servicios urbanos }\end{array}$ & + & + & + \\
\hline 10. Aumento en costo de la vivienda & + & + & + \\
\hline $\begin{array}{l}\text { 11. Desadecuación de la regulación del uso } \\
\text { del suelo }\end{array}$ & + & + & + \\
\hline
\end{tabular}

Norı: Valores (+) alto; (+-) medio; (-) hajo

El caso de Costa Rica es muy difícil de precisar, al no existir una delimitación por ley del Área Metropolitana. Basándonos en estudios de la CEPAL, se estimó que su población era de alrededor de un millón de habitantes en 1990, incrementándose a 1.27 millones en el año 2000 . La extensión de la mancha urbana se calculó en $95.3 \mathrm{~km}^{2}$ en 1999 (FUDEU, 1999), habiéndose incrementado en un $80 \%$ desde 1988. Al igual que en San Salvador, se trata de un crecimiento discontinuo, una especie de agregación de centros poblados antiguamente dispersos en el Valle Central. Pero lo que peculiariza a esta aglomeración urbana es que, en la Región Metropolitana se encuentran localizadas cuatro de las seis principales ciudades del país, no mediando entre ellas distancias mayores de veinte kilómetros. 
Panamá tenía, en 1980, 634 mil habitantes, sumando 980 mil en el año 2001. A diferencia de las dos ciudades anteriores, muestra una importante proporción de construcción en altura en los tres corregimientos del centro de la ciudad, alcanzando densidades mayores a las 2,500 personas por hectárea, duplicándose la altura promedio de los edificios, mientras continúa expandiéndose la ciudad en barriadas de viviendas de baja altura, destinados a los sectores de bajos ingresos en dirección al aeropuerto. La interrogante que surge en este caso es el impacto en este modo de ocupar el territorio que tendrá la utilización del área revertida del canal, cuya extensión, de inmediata posibilidad de urbanización, prácticamente supera la de la ciudad actual misma.

\section{Partiendo de las vulnerabilidades urbanas para avanzar en el análisis de los riesgos urbanos}

Es evidente que cualquiera que sea la modalidad de urbanización, ésta implica una relación entre la sociedad y el territorio que, inevitablemente, genera riesgos de distinta índole y distinto grado, pero también es claro que hay modos y umbrales de urbanización que provocan más riesgos y de diferente carácter que otras.

Esta afirmación, que parece trivial, no lo es si se incorporan factores que van más allá de los aspectos físicos (que algunos llamarían naturales), como la deforestación, el nivel de modificación de la lopografía, la densidad poblacional u otros, y se articulan a factores sociales, económicos, políticos o culturales. Bastan algunos ejemplos: la existencia o no de instituciones responsables del desarrollo urbano y el tipo de normativa urbana existente; el nivel de organización social y el tipo de relaciones predominantes de gobernabilidad urbana; el capital social o las percepciones que predominan sobre la ciudad; etc.; para no hablar de cuesliones más obvias como el grado de pobreza urbana o la segregación socioespacial.

El desafío que se presenta es, entonces, el establecimiento de las relaciones de causalidad entre los modelos de urbanización y los procesos de generación de riesgos que éstos implican. Este análisis, aún muy poco elaborado, pasa, en nuestra opinión, por una revisión crítica de lo que se ha llamado modelos de urbanización, en los que han predominado el enfoque demográfico-economicista, por un lado, y el enfoque político-culturalista, por el otro, sin lograr en la mayoría de las veces articularse en una visión integral. No pareciera tampoco que las visiones ambientalistas, a pesar de su pretensión holística, hayan logrado esta integración.

Pero este desafío implica también avanzar mucho más en el análisis de las causas de generación de los riesgos, particularmente en los ámbitos urbanos. Es decir, colocarse en el punto opuesto al desencadenamiento de los desastres, como se trata de expresar sintéticamente en el siguiente esquema: 


\section{GRÁFICO 1}

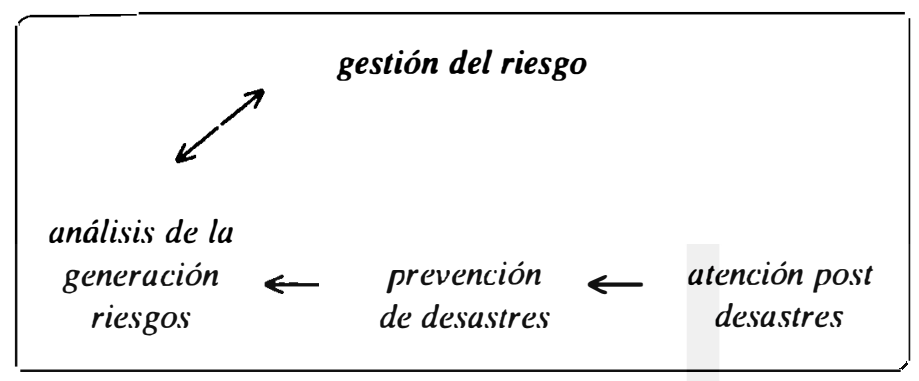

Por otra parte, y en mayor medida de lo que ocurre en el ámbito rural, en las ciudades tiene una singular importancia, y pasa muchas veces desapercibida, la generación constante de factores de riesgo menores, los que se caracterizan por:

a) Un período de conformación largo y acumulativo, íntimamente relacionado con las características del modelo de expansión urbana. El actual patrón de ocupación progresiva y descontrolada de tierras con vocación agrícola en el Área Metropolitana de San Salvador (AMSS), que se inició desde la década del setenta, constituye un buen ejemplo, siendo una de cuyas manifestaciones el deslizamiento de Montebello, ocurrido en 1982, que causó muertes y cuantiosos daños materiales.

b) Sus efectos son constantes y generalmente de pequeña magnitud, pero causando a mediano y largo plazo igual o mayores costos económicos y sociales que las grandes catástrofes (Blaikie et al, 1996).

c) Sus causas y efectos están vinculados a ámbitos regionales que superan el espacio urbano. La contaminación del aire, por ejemplo, rebasa el ámbito de la ciudad (Metzger, 1996).

Por los factores anteriores, la generación de riesgos ambientales urbanos no es siempre percibida en toda su magnitud (Barranqué et Kalaora, 1994). La población y las entidades gubernamentales no los consideran como una cuestión de vida o muerte. Pareciera que se aceptan como parte de la vida cotidiana.

Este trabajo trata precisamente de caminar en este sentido, examinando el caso de San Salvador en el próximo punto. Para tratar de contribuir al análisis de las causas generadoras de riesgos urbanos, partiremos, no de las amenazas, sean estas naturales o antrópicas, sino que tomaremos como eje del análisis las distintas formas de vulnerabilidad urbana. Al respecto, se han distinguido seis fuentes de esta vulnerabilidad (Lavell, 2000):

a) Concentración, densidad y centralización de personas y actividades

b) Complejidad e interconectividad de los procesos 
c) El peso de la informalidad y de la ciudad ilegal

d) La degradación del medio ambiente

e) La debilidad polílica e institucional

f) La falta de participación social en la polílica y la planificación

\section{En la mayoría de las ciudades latinoamericanas, persiste una prof unda fractura entre la ciudad legal, regulada, y la ciudad ilegal, la informal.}

La concentración de la población, pero, especialmente, de actividades que producen nuevos tipos de amenazas urbanas, muchas de ellas de tipo lecnológico, sumadas a la creciente complejidad e interconectividad de los procesos económicos y sociales, ha hecho que se produzca una agregación de niveles de vulnerabilidad antes pequeños, pero que sumados se convierten en situaciones de vulnerabilidad de gran envergadura.

Esto está vinculado, y se ve potenciado, por la debilidad de la legislación, del marco regulatorio y de las instituciones responsables del desarrollo urbano. La falta de participación de las organizaciones ciudadanas en este proceso termina de acentuar las distintas manifestaciones de vulnerabilidad urbana.

Especial impacto tiene lo anterior en otra fuente generadora de vulnerabilidad, el deterioro del medio ambiente en las ciudades. Aunque esta cuestión ha pasado a ocupar una posición central en las agendas de las políticas nacional y urbana, aún hace falta avanzar mucho más en el conocimiento de sus causas y posibles soluciones para reducir este deterioro.

Por otra parte, en la mayoría de las ciudades latinoamericanas, persiste una profunda fractura entre la ciudad legal, regulada, y la ciudad ilegal, la informal. Esta separación es también una enorme fuente generadora de vulnerabilidad que, afectando anteriormente, sobre todo, a los sectores sociales más pobres, por la creciente interconectividad de los procesos se va extendiendo a todo el conjunto social urbano.

Los planteamientos anteriores conducen a la cuestión clave de la construcción de indicadores de generación de riesgos urbanos.

\subsection{La construcción de indicadores de generación de riesgos, un instru- mento indispensable para la gestión del riesgo en las ciudades}

En el camino por evitar la tentación antes mencionada e incidir efectivamente, Aún en el reciente trabajo que se ha iniciado, de construcción de indicadores urbanos en América Latina, por iniciativa del Programa de Gestión Urbana (PGU- 
HABITAT/ PNUD, 2000), predomina una visión en que los indicadores de riesgo, que realmente se limitan a la prevención de desastres, son un aspecto más, sumado sin articulación a otros más, en una concepción muy tradicional.

Este trabajo, creemos, sólo puede llevarse a cabo con una activa participación de los investigadores sociales que han estado trabajando ampliamente esta problemática.

\section{Creemos que es indispensable realizar un trabajo apenas esbozado: la construcción de indicadores de generación de riesgos urbanos.}

Ante la insuficiencia señalada se propone la incorporación de indicadores de generación de riesgos por la modificación de los usos del suelo debido a cuatro procesos: los cambios ambientales, las transformaciones en la economía, los cambios tecnológicos y las modificaciones socio-institucionales:

Las transformaciones en estos procesos tienden a generar nuevos riesgos y potenciar los existentes. La construcción de los indicadores se haría alrededor de los procesos anteriores. El esquema siguiente trata de visualizar esta idea:

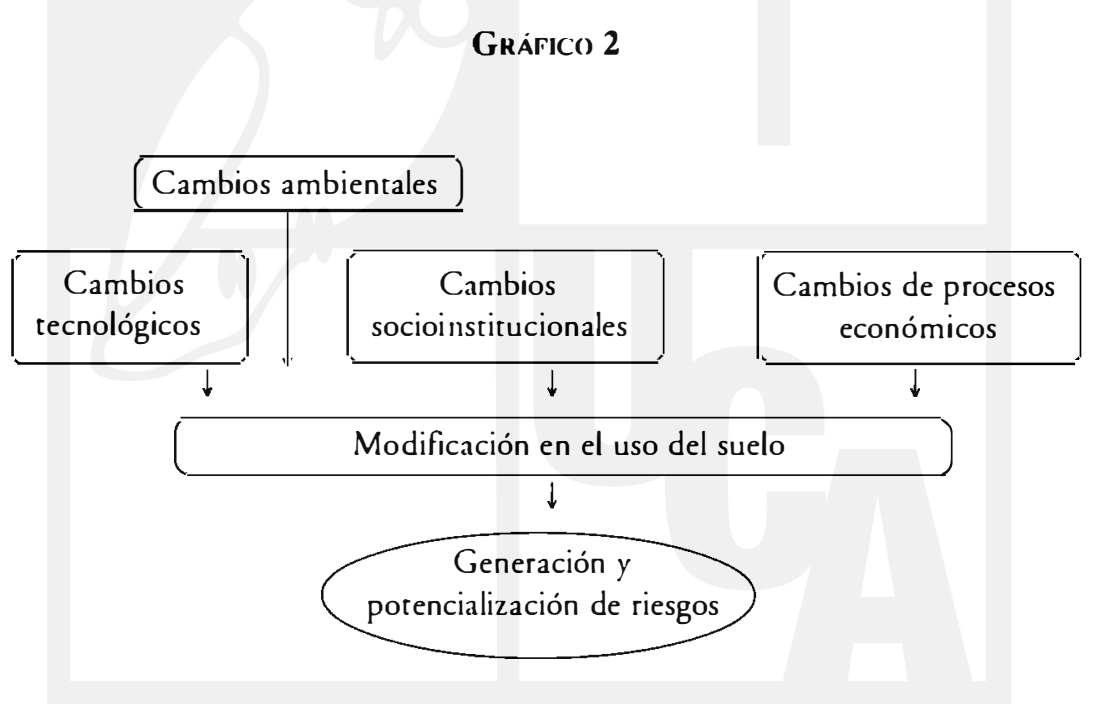

Tratemos de plantear, con un carácter preliminar, ejemplos de cuáles serían los indicadores de generación de riesgos, base indispensable para poder pensar en la gestión de riesgos. 


\section{La generación de riesgos ambientales no es siempre percibida en toda su magnitud. Pareciera que se aceptan como parte de la cotidianidad.}

\section{Hacia la construcción de indicadores de generación de riesgos en el Área Metropolitana de San Salvador}

Sobre el Área Metropolitana de San Salvador existe información muy dispersa y fragmentada, poca sobre aspectos económicos y elaborada en general, siguiendo los patrones usuales, a pesar de intentos hechos en los últimos años para avanzar en su sistematización y utilizar nuevos enfoques para fines específicos (Lungo y Oporto, 1994; Umaña, 1996; PLAMADUR, 1997; PRISMA, 1997; OPAMSS, 2000).

De estos datos queremos, como ejemplo, mostrar algunos que, con la lógica expuesta anteriormente, pueden contribuir a la construcción de indicadores de generación de riesgos urbanos. Así, puede verse la relación entre densidad poblacional y porcentaje del suelo urbanizado para dos municipios del AMSS:

- Cuscatancingo: $192 \mathrm{~h} / \mathrm{km}^{2}$ y $83 \%$ del suelo urbanizado.

- Tonacatepeque: $27 \mathrm{~h} / \mathrm{km}^{2}$ y $20 \%$ del suelo urbanizado.

Rápidamente observamos que el primero está altamente urbanizado a diferencia del segundo, que es aún predominantemente rural. A primera vista, parecería obvio que el primero presente mayores riesgos urbanos. Sin embargo, un análisis dinámico mostraría que la generación de riesgos es mayor al estar sometido a un acelerado proceso de urbanización sin el nivel de infraestructura con que se urbanizó el primero y con modificaciones en la topografía que originarán riesgos de nuevo tipo y dimensión por ser los proyectos que se están edificando en el último de gran tamaño. Lo anterior reafirma la idea de que lo importante es el análisis de los procesos más que de los datos estáticos, por muy válidos que estos sean.

\section{Lo importante es el análisis de los procesos más que de los datos estáticos, por muy válidos que estos sean}

Otro ejemplo de distinto tipo: generalmente se presentan datos sobre cobertura de servicios, por ejemplo, acceso a redes colectoras de aguas negras, cuando es más importante señalar que la red principal del AMSS fue diseñada y construida con un horizonte para el año 1982 y que el crecimiento urbano ha ido a un ritmo mucho mayor que las ampliaciones de la red que se han realizado

Expansión de las ciudades en Centroamérica 


\section{Cuadro 5 \\ Lista de indicadores correspondientes a las veinte áreas de compromisos clave de la Agenda Hábitat}

\author{
Capitulo I: Vivienda \\ 1. Posibilitar la se'guridad de la tenencia \\ Indicador I: regimenes de tenenciat \\ Indicador 2: desalojos \\ 2. Promener el derecho a vivienda adecuada \\ Dato cualitativo I: derecho a vivienda \\ Indicador 3: cocficiente cntre precio c ingresos de la vi. \\ vienda \\ 3. Proporcionur igualdad de acceso a la herra \\ Indicador 4: acticiente entre procio de la licra ce ingrexses \\ 4. Promover igualdad de accessos a creditos \\ Indicador 5: Préslamos con hipoleca y sin hipolecil \\ 5. Promover el acceso los servicios heisicos \\ Indicador 6: acceso al agua \\ Indicador 7: conexiones domiciliarrias
}

\section{Capilulo 2: Desarrollo social y erradicación de la pobreza}

6. Promover la igualdad de oportunidales para mon vida sama y segura

Indicador 8: mortalidad de niños menores de 5 años Indicador 9: lasus de delincucncia

Dalos cualilativo 2: violencia urbana

7. Promover la integración social y upoyar a los grupos desfavorecidos

Indicador 10: hogares probres

8. Promover la igualdad de género e'l el desurrollo de a.sentamicntos urhanos

Indicador II: brechas entre hombres y mujeres

\section{Capitulo 3: Ordenamiento ambiental}

9. P'romover uno esiructura geogrificamente extuilitiadu de los asentamientos humanos Indicador 12: crecimiento de la población urbana

10. Administrar el suministro y demenda de agua de forma eficuz

Indicador 13: Consurno de agua

Indicador 14: Prccio dcl agua

11. Reducir la comaminacion en zonas urhanas Indicador 15: Contaminación atmosféricil Indicador 16: Aguas rcsiduales Iratadas Indicador 17: Eliminación de dcscchos sólidos

12. Prevenir los desastres y reconsiruir los asentamicintos Dato cualialivo 3: Prevención de desasitres $\mathrm{c}$ insirumenlos de miligación

13. Promover sistemas de transporte eficaces y ambientulmente racionales

Indicador I8: Ticmpo de Iraslado

Indicador 19: Medios de Iransporte

14. Prestar apoyo para preparar y aplicar planes ambientales lacules e inicierrivas de la Agenda 21 locules. Indicador cualitalivo 4: Planes ambientiles locales

\section{Capítulo 4: Desarrollo econcimico}

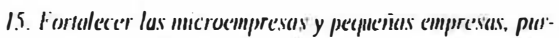
ricularmeme las exsablecidas por imrjeres

Indicidor 20): empleo informal

16. Fomentar las ascriaciomes de los secioses publico y prirado y estimular las opormunidades de empleon productiros Dalo cualiitiativo 5 : asociaciones publicas y privadats Indicadar 21: produclo urbano Indiciador 22: desempleo

\section{Capilulo 5: Gobernabilidad}

17. Promoier la descentralancioin y formalecer las autorida des locules

Dalo cualitalivo 6: nivcl de desteniralización

18. Fonenter y apoyer la pxuricipacien y el compromiso crivico Dato cualitativo 7: parlicipacionn ciudadana en las decisioncs importanics sohrc la planificación

19. Cinruntizar la adminisirucion transparente, responsuble y eficaz de pueblos, cindades y zonos metropolitanas: Dalo cualitalivo 8 : Transparencia y responsabilidad Indicador 23: Ingresos y gasios de los gohicrnos Inciles

\section{Capitulo 6: Cooperación internacional}

20). Liortalecimiento de la cooperación internacional y las asociaciones:

Dalo cualialivo 9: Impulsar la coupcracion y lass asociaciones internacionalcs 


\section{Cuadro 6}

Indicadores de generación de riesgos

\begin{tabular}{|c|c|}
\hline EJE DE ANÁLISIS & INDICAUORES \\
\hline \multirow{8}{*}{$\begin{array}{l}\text { MODIFICACION } \\
\text { EN EL USO } \\
\text { DEL SUELO }\end{array}$} & CAMBios ambientales \\
\hline & $\begin{array}{l}\text { - Porcentaje deáreas cubiertas de vegetación (regula- } \\
\text { ción hídrica y del aire). } \\
\text { - Porcentaje de áreas impermeabilizadas (incidencia } \\
\text { en el volumen de escorrentía superficial). } \\
\text { - Número y tipo de cortes y rellenos. }\end{array}$ \\
\hline & CAMbios en los PROCESOS ECONÓmiCos \\
\hline & $\begin{array}{l}\text { - Índice de concentración de industrias contaminan- } \\
\text { tes del aire y del agua. } \\
\text { — Índice de concentración de actividades comerciales } \\
\text { (generadoras de desechos y de concentración de trá- } \\
\text { fico). } \\
\text { - Concentración poblacional en áreas de riesgo. }\end{array}$ \\
\hline & Cambios tecnológicos \\
\hline & $\begin{array}{l}\text { - Grado de modernización de los procesos construc- } \\
\text { tivos (en términos de reducción de riesgos). } \\
\text { - Procesos de modernización de los sistemas de trans- } \\
\text { porte y de infraestructura vial. } \\
\text { - Cobertura y tipo de los sistemas de drenaje. }\end{array}$ \\
\hline & CAMBIOS SOCIOECONÓMICOS \\
\hline & $\begin{array}{l}\text { - Nivel de organización social respecto a riesgos. } \\
\text { - Grado de desarrollo de la institucionalidad respon- } \\
\text { sable de riesgo. } \\
\text { - Grado de incorporación del factor riesgo en los pla- } \\
\text { nes de desarrollo y el marco normativo. }\end{array}$ \\
\hline
\end{tabular}


desde ese año. Tomemos de nuevo datos para los dos municipios anteriores. En 1992, el $59 \%$ de las viviendas de Cuscatancingo estaban conectadas a esta red, y sólo el $41 \%$ en Tonacatepeque. Estos datos se deben, sin embargo, relacionar con la forma que asume la organización, el sistema de cuerpos de agua o que-. bradas y las actividades económicas que se realizan en sus territorios.

Sólo así, datos genéricos como que en los últimos treinta años el área urbanizada del AMSS ha duplicado su extensión, o que el $70 \%$ del caudal de aguas negras se vierte a las quebradas o ríos, adquieren un sentido para análisis de procesos específicos como el que nos ocupa.

Con esta finalidad, hemos tratado de avanzar un poco más en la propuesta general formulada en el cuadro anterior, proponiendo veinte indicadores que priorizaríamos para el estudio de la generación de riesgos urbanos:

\section{Cuadro 7}

Priorización para la construcción de indicadores de generación de riesgos urbanos en el caso del AMSS

\begin{tabular}{|c|c|c|c|c|}
\hline \multicolumn{2}{|r|}{ INDICADOR } & TIPO & INFORMACIÓN & UNIDAD \\
\hline 1. & Area urbanizada & Cuantitativa & Es necesario actualizarla & $\mathrm{Km}^{\prime}$ \\
\hline 2. & Área a urbanizar & Cuantitaliva & Se debe proyectar lendencialmente & $\mathrm{Km}:$ \\
\hline 3. & Área no urbanizable & Cuanlilaliva & Prever usos & $\mathrm{Km}:$ \\
\hline 4. & Área impermeabilizada & Cuanlilativa & Es necesario construirla & $\mathrm{Km}:$ \\
\hline 5. & Área cubierla de vegelación & Cuanlilativa & Proyeclar modificación & $\mathrm{Km} \mathbf{m}^{\prime}$ \\
\hline 6. & Áreas de corles & Cuantilativa & Es necesario consiruirla & $\mathrm{Km}$ lineales \\
\hline 7. & Áreas de rellenos & Cuanlilativa & Es necesario consiruirla & $\mathrm{Km}^{\prime}-$ \\
\hline 8. & Industrias conlaminantes & Cuantilativa & Es necesario construirla & Número \\
\hline 9. & Ceniros de comercio & Cuanlilativa & Es necesario construirla & Número \\
\hline 10. & Infraesiruclura vial & Cuantilativa & Es necesario actualizarla & $\mathrm{Km}$. lineales \\
\hline 11. & Puenles y pasos a nivel & Cuanlilativa & Es necesario actualizarla & Número \\
\hline 12. & Bóvedas & Cuantitativa & Es necesario actualizarla & Unidades \\
\hline 13. & Drenajes aguas Iluvias & Cuantitativa & Es necesario aclualizarla & $\mathrm{Km}$. lineales \\
\hline 14. & Drenajes aguas negras & Cuantitativa & Es necesario actualizarla & $\mathrm{Km}$. lineales \\
\hline 15. & Viviendas en zonas de riesgo & Cuanlitaliva & Es necesario aclualizarla & Unidades \\
\hline 16. & Planes de prevención & Cualitativa & Es necesario aclualizrla & $\begin{array}{l}\text { Análisis de } \\
\text { conlenido }\end{array}$ \\
\hline 17. & Instituciones encargadas & Cualitativa & Es necesario aclualizarla & $\begin{array}{l}\text { Anàlisis de } \\
\text { contenido }\end{array}$ \\
\hline 18. & Inversión en prevención & Cuantitativa & Es necesario construirla & $\begin{array}{l}\text { Unidad } \\
\text { monetaria }\end{array}$ \\
\hline 19. & Participación en prevención & Cuantilativa & Es necesario consiruirla & Número \\
\hline 20. & $\begin{array}{l}\text { Participación ciudadana en } \\
\text { prevención }\end{array}$ & Cualitativa & Es necesario construirla & $\begin{array}{l}\text { Análisis de } \\
\text { conlenido }\end{array}$ \\
\hline
\end{tabular}


El desafío metodológico consiste en pasar a reconstruir analíticamente procesos, partiendo de información - consistente tanto en datos cuantitativos como valoraciones cualitativas - obtenida para un momento dado. Un recurso utilizado en un trabajo anterior (PRISMA, 1997), la construcción de escenarios, mostró su utilidad, tanto para el análisis como para exponer sintéticamente las tendencias y los cambios que se provocarían al intervenir sobre estos procesos. Lo anterior no es nada nuevo. Se ha utilizado mucho en los estudios políticos y econométricos. Lo interesante es su utilidad para el análisis de la generación de riesgos urbanos, lo que permitiría dar un salto en la gestión del riesgo en las ciudades.

Evidentemente, en este trabajo sólo es posible hacer planteamientos preliminares sobre esta cuestión, y sería necesario realizar investigaciones en profundidad para desarrollar el enfoque metodológico esbozado.

\section{La participación de los gobiernos locales y la ciudadanía en la gestión de los riesgos urbanos}

En trabajos anteriores hemos partido de que la gestión urbana implica cuatro procesos íntimamente ligados (Lungo y Pérez, 1991):

- La planificación urbana

- La regulación

- La administración del desarrollo de la ciudad (uno de cuyos instrumentos es el marco regulatorio)

- La inversión urbana (tanto pública como privada)

Una gestión de tipo integral implica una integración de estos procesos, lo que en muy pocos casos ocurre.

Lo anterior hace que, también en los pocos casos en que se ha comenzado a incorporar la gestión de riesgos en el desarrollo de las ciudades, estos esfuerzos se segmenten entre estos cuatro procesos, y podríamos plantear que donde se ha avanzado más es quizás en el campo de la regulación (por ejemplo, introduciendo normas relativas a la construcción en las llamadas zonas de riesgo).

Dada la disociación imperante entre estos cuatro procesos, lo que está relacionado con la forma en que está estructurado el aparato gubernamental y con la manera en que ser formulan las políticas públicas, pareciera difícil que la introducción de la gestión de riesgo pueda hacerse de forma integrada. Creemos que la vía es identificar, en cada caso concreto, cómo se relacionan estos procesos y cuáles predominan, para priorizar los esfuerzos sobre estos últimos y no desperdiciar recursos en esfuerzos de carácter genérico.

En el caso de las ciudades centroamericanas, donde la planificación es muy débil, el marco regulatorio (generalmente obsoleto), tiene poca aplicación, y la institucionalidad administradora del desarrollo urbano contradictoria y descoordi- 
nada (Lungo, 1998), podría plantearse que el énfasis debería dedicarse a incidir en la introducción de la gestión de riesgos en los procesos de inversión. Esto no es, sin embargo, tan simple. Sin un marco regulatorio modemo que combine adecuadamente normas e incentivos es difícil incidir positivamente en los procesos de inversión, lo que nos vuelve a remitir, al menos, a la importancia de contar con un marco institucional capaz de conducir estos procesos.

Surge entonces la tentación, como en el caso del medio ambiente, de intentar que la gestión de riesgos contribuya a la integración de funciones e instituciones dispersas, peligro sobre el que queremos llamar la atención para no pretender llevar la gestión de riesgos más allá de sus posibilidades de incidencia real.

\section{Surge la tentación, como en el caso del medio ambiente, de intentar que la gestión de riesgos contribuya a la integración de funciones e instituciones dispersas.}

En el momento actual estamos en presencia, en muchos países centroamericanos, de una triple división entre la gestión urbana, la gestión ambiental y la gestión de riesgos. La primera está comenzando a recuperar el campo perdido desde los años 70 del siglo XX, estimulada por dos procesos aparentemente contradictorios: la globalización y la descentralización. La segunda tiene una fuerte presencia desde los años 90 , debido a la búsqueda de opciones de desarrollo sostenible. La tercera ha comenzado a estructurarse, aunque aún es muy débil, a raíz de los efectos de la tormenta Mitch y los múltiples programas que se han promovido para la recuperación de las zonas afectadas.

Podríamos afirmar, entonces, que la incorporación de la gestión de riesgos en la gestión urbana pasa por la integración de ambas con la gestión ambiental.

A esta dispersión institucional y de las políticas públicas se suma la insuficiencia del análisis y de la información, que responden a estas visiones fragmentadas. Adicionalmente, en el caso de las principales ciudades centroamericanas, estamos ante aglomeraciones urbanas de dimensión metropolitana, lo que agrega complejidad y dificultades adicionales a su gestión. Veamos, a continuación, algunos de estos problemas. 


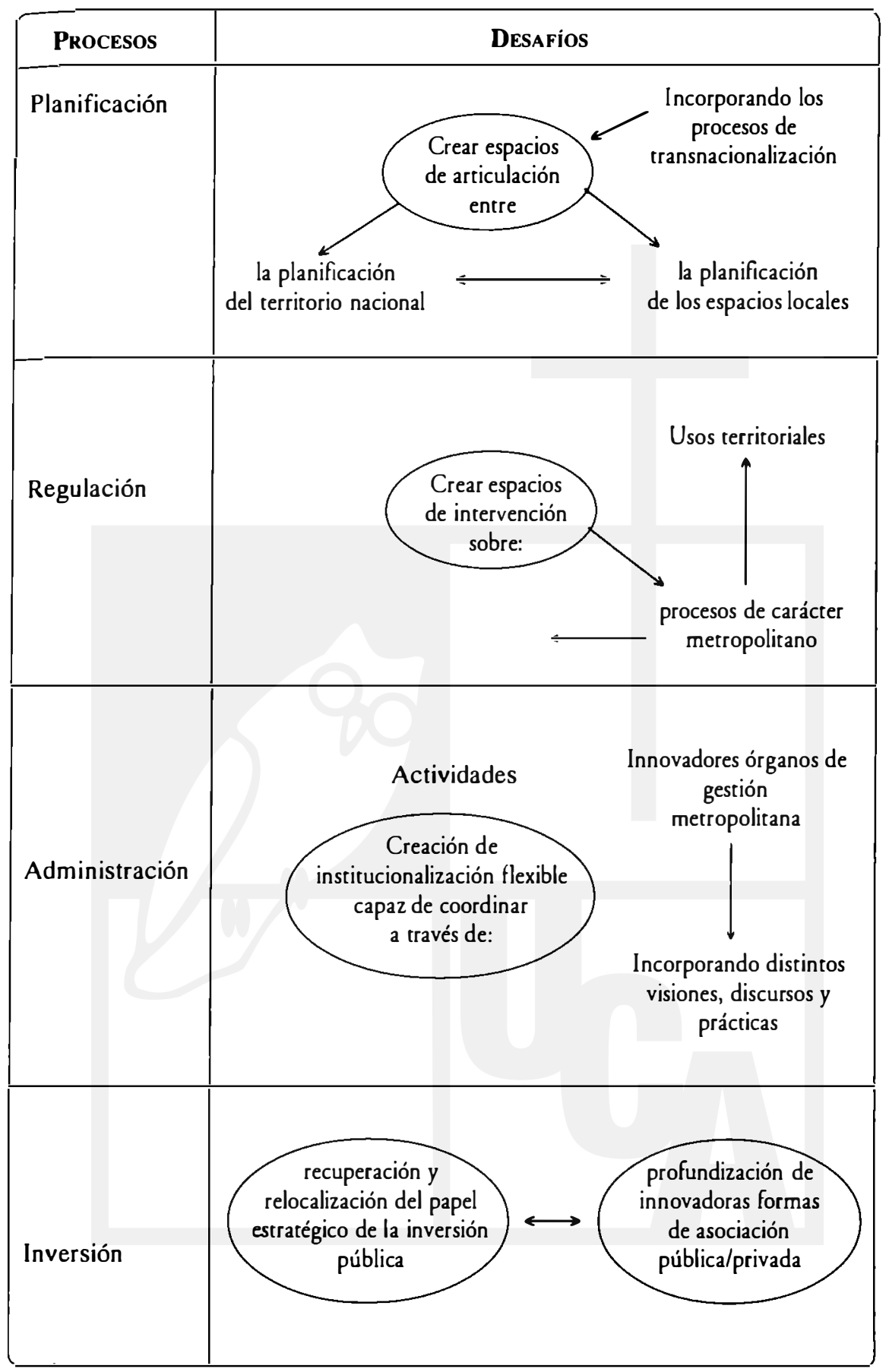




\section{Gráfico 4}

Principales desafios y actores de la gobernabilidad metropolitana

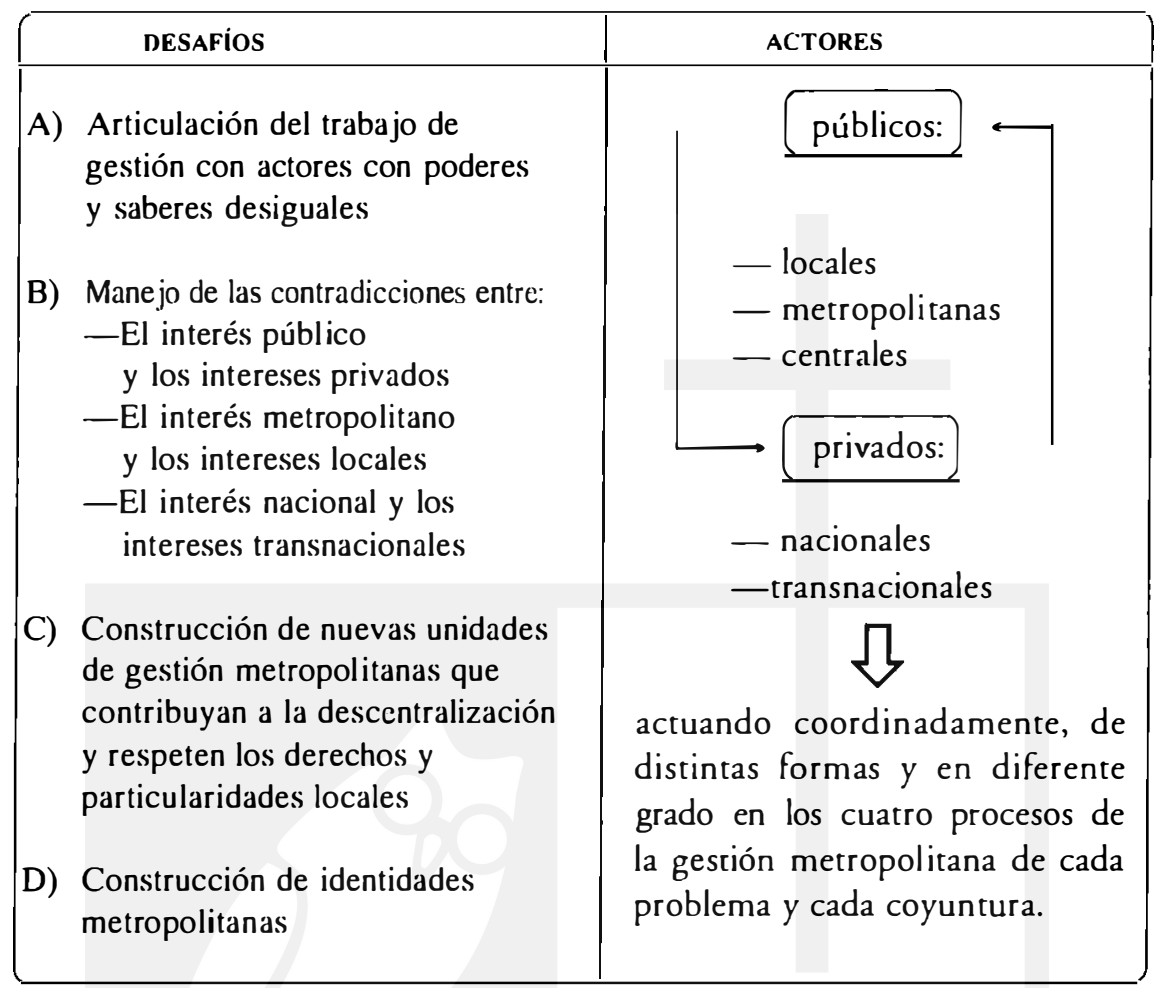

Estas reflexiones sobre la gestión de las ciudades permiten establecer dos conclusiones esenciales para la problemática abordada en este trabajo:

1') Se debe caminar hacia la inserción de la gestión de riesgos en la gestión urbana.

Como indicábamos anteriormente, hay que evitar la disociación entre la gestión del desarrollo de las ciudades y la gestión de los riesgos ambientales. Permitirlo es debilitar la necesaria intervención del Estado y las organizaciones ciudadanas en el manejo del desarrollo urbano y, por lo mismo, crear las condiciones para la generación de nuevos riesgos urbanos y el potenciamiento de los ya existentes con todas las consecuencias que podemos imaginar.

2) Los ejes de trabajo específicos a priorizar son: La incorporación de la gestión de los riesgos a la planificación y al marco regulatorio urbanos, y la promoción de la participación de los distintos actores. 
Las propuestas anteriores deben especificarse al nivel urbano para efecto de elaborar proyectos específicos en este ámbito. La mejor estralegia, creemos, es insertar esta problemática en los planes en ejecución o elaboración. Así, por ejemplo, incorporar en los Planes de Desarrollo Urbano la gestión de riesgos, especialmente a través de la planificación de acciones que reduzcan la generación de riesgos, lo cual implica realizar estudios específicos que deben orientarse por planteamientos metodológicos como los hechos en páginas anteriores.

En torno a la gestión, deberían crearse unidades especializadas en las oficinas encargadas del desarrollo urbano, las que serían responsables de implementar las regulaciones tendientes a la reducción o para minimizar la generación de riesgos.

Habría que pensar también en explorar la creación de un fondo particular para la gestión de riesgos para las ciudades, fondo que debería ser alimentado, en parte, por las actividades generadoras de estos.

Finalmente, más que promulgar leyes de carácter general que tienen poca operatividad, es indispensable repensar radicalmente el marco regulatorio urbano, incorporando instrumentos modernos, tanto de orden restrictivo como incentivos, para los aspectos de urbanización y construcción y también para las distintas actividades que se realizan en las ciudades.

\section{REFERENCIA}

- Barraqué, Bcrnard ct Kalaora, Bcrnard (1994): Introduction. Le risque environnemental et son vécu, en Espaces et Sociétes $\mathrm{N}^{\circ} 77$, ćd. Anthropos, París.

- Blaikic, Picrs; Cannon, Tcrry; Davis, lan; Wisner, Ben (1996): Vulnerabilidad. El entorno social, político y económico de los desastres.

- Davis, Mikc (1998): Ecology of Fear. Los Angeles and the Imagination of Disaster, Vintagc Books, Ncw York.

- Fcrnándcz, María Augusta (1996): Ciudades en riesgo, La Rcd/ USAID, Lima.

- FUDEu (1999): Tendencias del crecimiento urbano en el área de estudio para el Plan Maestro de Desarrollo Urbano de San .José, mi meo, San Josć.

- Huct, Lionel (1999): Diagnóstico del drenaje urbano en el Área Metropolitana de San Salvador, BID-OPAmss, San Salvador.

- Instituto de los Ingcnicros Civilcs c ITDG, Pcrú (1999): Megaciudades. Reduciendo la vulnerabilidad a los desastres, Lima.

- Lavcll, Allan (2000): Desastres urbanos: una visión global, en Momentos, año 15, númcro 5, ASIEs, Guatcmala.

- Loti e Associati (2000): Diagnóstico, factibilidad y diseño final del sistema secundario de drenaje de aguas lluvias en cuatro zonas criticas del Área Metropolitana de San Salvador, MOP, San Salvador.

- Lungo, Mario (1998): Gobernabilidad urbana en Centroamérica, F.ACsO-GURI, San Josć. (2000): Environmental Risks and Reconstruction Post-Mitch. Sustainable Urban

Development in Central America, ponencia presentada a la conferencia organizada por cl Centro Woodrow Wilson, Guatemala.

Expansión de las ciudades en Centroamérica 
—— y Pérez, Mariam (1991): Gestión urbana: algunas cuestiones teóricas, en ESTUdios sociales Centronmericanos, $N^{\circ} 55$, San José.

Salvador

y Oporto, Francisco (1994): San Salvador. Estadisticas básicas, flacso, San

- y Pohl, Lina (1996): Las acciones de prevención y mitigación de desastres en El Salvador: Un sistema en construcción, en De terremotos, derrumbes e inundaciones, M. Lungo y S. Baires (compiladores), rUNDE, San Salvador.

et al (1998): Estrategia ambiental para la región metropolitana de San Salvador, PRISMA, San Salvador.

—_ y Baires, Sonia (1998): Hábitat popular urbano y riesgos ambientales, Universidad de Lund/ Universidad Mayor de San Simón, Cochabamba.

- Metsger, Pascale (1996): Medio ambiente urbano y riesgos. Elementos de reflexión, en Ciudades en riesgo, M. A. Fernández (compiladora), LA RED/USAID, Lima.

- opamss, Oficina de Planificación del Área Metropolitana de San Salvador (2000): Propuesta para el Programa Cities Alliance.

-_- (1999): Plan de rescate del Centro Histórico de San Salvador.

(2000): Indicadores urbanos del Área Metropolitana de San Salvador.

- Portes, Alejandro y Lungo, Mario (1992): Urbanización en Centroamérica, fLACSO, San José.

- PrISMa (1997): Perfil ambiental de la región metropolitana de San Salvador, PRISMAAID, San Salvador.

- Programa de Gestión Urbana para América Latina, habitat-pnud (2000): Indicadores urbanos Estambul+5. Guia, Quito.

- Rodrigue, François (1999): Los efectos del Mitch en el municipio de Delgado, área metropolitana de San Salvador, opamss, San Salvador.

- Uribe, Álvaro (1989): La ciudad fragmentada, CELA, Panamá.

- Viceministerio de Vivienda y Desarrollo Urbano (1996): Indicadores urbanos y de vivienda, vMVDU/GTz.

(1997): Plan de Desarrollo del Área Metropolitana de San Salvador ampliada. 